\title{
Tire Pressure Monitoring System
}

\author{
Dr.Gayatri M.Phade, Sandip Foundation (SITRC), Nashik India, gayatri.phade@sitrc.org
} Aniket Dinesh Kulkarni, Sandip Foundation (SITRC), Nashik India, dkulkarnianiket@gmail.com.

ABSTRACT - Tire pressure monitoring device/system (TPMS) is an electronic device that monitors the air strain of an vehicle tire and indicates the pressure to the driver.Upkeep of tire strain in automobile is vital owing to the reality depletion within the strain of tire ends up in diminished combustible potency and future scratch in tires which ends up in substitution of tires fairly again and again. In this paper the used conception relies upon eliminating above problems and observing those facts continuously using pressure sensor with the help of some devices and wireless modules such as zigbee or rf-transreciever and also STM32 on-chip computer. A different mouth is distended other than conventional muzzle within that device is found which communicate via wireless apparatus and obtained with the help of the wireless module such as zigbee gift out of doors and also the signal is been dispatched to on-chip computer which computer screen units and unveil the strain name (name of tire) and actual strain on a screen monitor which notify the person to fill the air in tire. With the use of this system the user of the vehicle can eliminate the wheel misalignment and supply protection to the vehicle.

Keywords --TPMS (Tire Pressure monitoring System), ZigBee, Pressure Sensor.

\section{INTRODUCTION}

\section{1] MOTIVATION:}

Every year, many accidents took place and for certain cases, injuries are as a result of beneath-inflated tires. Under inflated tires ought to promote to troubles together with blowouts, decreased tire lifestyles, and handling. The great of jogging the tires at the specified strain allows provide proper car dealing with (as a consequence, reducing the chance of accident) at the same time as heading off untimely tire put on. The right pressure for a vehicle is properly- stated at the tire data label or tire placard located on a door area or door jamb, or within the glove-box door. The label additionally lists maximum load and tire length (together with spare). Below inflated tires wear on the outsides of the tread. Additionally, the tires flex excessively which produces extra warmness and faster put on.

Over inflation causes the center of the tread to put on. The tire cannot flex usually and thisputs pressure at the sidewalls and plies. It isn't handy to regularly test the tire strain the usage of pressure gauge. For lengthy adventure, tire pressure can also range on occasion due to load, road irregularities, and temperature. As a result, one could not likely understand the condition of the tire and that had caused many tire blowouts specifically for heavy vehicles. Subsequently, TPMS is delivered. TPMS is an electronic device that observes and display units the air stress. The device alerts the motive force of the car of the air stress inside the tires by means of displaying the real strain or just a caution mild. Some of the car manufacturers already set up their very own TPMS on their automobiles.

\section{2] TYPES OF TPMS:}

TPMS categorized in 2 different sorts - Straight (direct(tpms)) and oblique (indirect-(tpms)). TPMS are given to aftermarket (manufacturing facility) degree as well as an OEM answer. The goal of a TPMS is fending off visitor's accidents, terrible fuel economic system, and elevated tire wear because of underneath-blown up tires via premature realization of a unsafe for the tyre.

- Direct TPMS

- In-Direct TPMS A] Straight TPMS:

Straight TPMS appoint stress detectors on each trundle, both inner and outside. The detectors bodily quantify the wheel strain in each wheel and record that into device or a correlate observe.A few gadgets also degree and vigilant hotness of the tyre as nicely. Such sort of apparatus can find out underneath- blown up in any mixture,for any tyre, simultaneously. Despite the fact that the structures differ in pass on alternatives, numerous tpms merchandise (each native apparatus manufacturer and after market) able to show synchronous tire compulsion at every position record whether or not now the car is transferring or standing idle. There are numerous extraordinary answers, however each of them should face the problems of subjection to antagonistic circumstances.A few detectors make use of a wi-fi power gadget just like that utilized in rfid label analyzing which gives the answer to the hassle of restricted accumulator lifestyles next to way of electromagnetic induction. This additionally will reverberate the density of information transmission as much as forty $\mathrm{hz}$ and lessen the detector heaviness which may be essential in car sport programs. 


\section{B] In-Direct TPMS:}

Oblique(In-Direct)TPMS do no longer use bodily strain detectors but quantify air force by observing solitary wheel revolving momentum and different alerts obtainable exterior of the wheel itself. Opening technology straight tpms structures are depend on totally at the fundamental that beneath- aerated wheels have a somewhat little broadness than a effectively aerated one.Those variations are quantifiable through the wheel pace detectors of abs/esc devices. Subsequent generation straight tpms also hit upon concurrent below-aerated in overall 4 tires utilizing range inspection of wheels, which may be found out in operating system the usage of superior sign action techniques. The range inspection depends on precept that sure,repetition of the tyre and disc assembly are noticeably responsive to the aerated strain. Those swings result are able to be observed via superior response of the wheel pace indicators. Modern straight tpms made up of firmware modules being embedded into the anti breaking system's assembly.

\section{LITERATURE REVIEW}

An evaluation of present tire strain tracking gadget us division of transit wheel- velocity primarily depend on TPMS because the web structures use wheel velocity to evaluate the spin radius of the tire, it became important that the tires had uniform tread put on, that the car changed into well burdened, and the tire strain were set at the recommended placard bloodless inflation strain even as bloodless. Earlier than trying out, each web TPMS became reset and calibrated for each automobile in line with the producer's endorsed method. Because the energetic spin radius of a tire changes considerably with pace, the structures have to be calibrated in a couple of pace tiers. If a car producer recommends a growth in inflation pressure to deal with a better load, web structures require recalibration. As an instance, if a car was to be tested at the gross automobile weight score at a particular tire strain, it becomes additionally calibrated in that nation.

\section{1] Proposed System:}

Evaluation of potential access factors this phase discusses the pros and cons of every of the capacity get admission to factors. Those get right of entry to points all talk wirelessly with the automobile can bus, which is why they had been chosen as the ability get entry to factors to be evaluated. The get entry to factors consists of TPMS, key fob, invehicle Wi-Fi connectivity, and GPS. Tire strain tracking system the tire strain tracking machine turned into considered as a capacity access point because of the huge amount of studies to be had on the topic. Numerous research were made available because the tire pressure sensors had been made mandatory for all vehicles pointing out that it is viable to send messages to the can bus thru these sensors. There are recognized methods of reading TPMS signals using software program defined radios, antennas, and floor acoustic wave (noticed) filters. Researchers in Rutgers College and university of South Carolina have accomplished spoofing already. The group theorizes that sending a mimicked signal to the can bus is probably feasible if the mimicked signal is stronger than the signal being dispatched through the tire strain sensor in the tires. Thru this approach they might be communicating with the can bus wirelessly. Tpms works with radio frequency at a frequency of $315 \mathrm{mhz}$, which the team can use the to be had software- described radio for, These sensors speak at once with the European which in turn communicates with the can bus, making those sensors a possible manner of reaching the project's cease purpose of sending a sign to the can bus. Inside the schematic under, one can see the layout of the TPMS communications between the sensors, the receiver and the show inside the dashboard of the vehicle.

\section{2] Other Reviews:}

- Mark Reiter and John Wagner learning the result of wheel strain on motor managing based totally at the automated automotive tire inflation machine. The opportunity to regulate car coping with via an automatic tire enlargement strain device able to modify chassis outcome. In this written paper, a motor's coping with conduct being observed for numerous belowenlargement wheel scenarios. The the fore wheel stress can be diminished to provide more under steer. A front tire strain may be reduction results in larger steering wheel angles, at the same time as back wheel strain discounts creates large motor skid gradient (less automobile firmness and better automobile over steer).Directional instability will become obtrusive with heaviness move toward back blended with backend wheel strain discount according to the growth in firmness gradient. Hereafter analytical study will observe the control techniques.

- Sadda. Mahendra, N. Amara nageswara rao, studied the effect of tire overload and inflation pressure on rolling loss and gas intake of vehicle. On this thesis, the effect of tire over load and inflation strain at the rolling loss and fuel intake is analyzed. The investigations are made on fashions on fashions of tire Skoda fast and ford classic. The analysis is carried out by means of applying the masses of automobile weight and human's weight. Whilst the auto is overloaded, additionally analysis is done. Modeling is completed in pro/engineer and evaluation is done in annoys. By evaluating the outcomes ford traditional tire is best that's getting less pressure values compared to Skoda tire inside the case of overloaded condition and the gas intake additionally extra for Skoda.

- Joao m. Serrano, jose o. Percent, rafael silva, studied the impact of liquid ballast and tire inflation strain on 
tractor overall performance. The paper offers the end result of evaluation of impact of liquid ballast and tire inflation strain on traction parameter and performance of an agricultural tractor under discipline operating situations, on dry sandy loam soil. It's far proven that there have been no considerable differences both in work fee in gas intake according to hectare among the tire inflation strain particular via the tractor synthetic and that is detailed by tire manufacturer.

- T. Smerda, j. Cupera, studied the tire inflation and its impact on drawbar traits and overall performance of zestful signs of a tractor set. The paper presents the effects of understand quantification divulge that lessen tire escalation of suitable tire sorts can better the drawbar attribute and therefore fuel utilization.It became visible that the dispense attribute, the tractor move substantial drawbar tug to the floor at the exact floor speed and underneath swollen stress. Huge shift drawbar tug approach an expand of drawbar energy and tractor overall outcome.

\section{SYSTEM DEVELOPMENTS}

\section{1] BLOCK DIAGRAM:}

There are two styles of not unusual tpms. One is wheelspeed based tpms. This gadget video display devices wheel pace signals through the wheel velocity sensor of abs tool. Tire diameter turns into large or small whilst high quality tire strain is simply too high or low, while wheel pace additionally correspondingly modifications. After which it'll provide an alarm if tire stress is absolutely too excessive or inadequate. Such gadget can't determine the situation of greater than tires being lack of gas on the equal time or velocity exceeding $100 \mathrm{~km} / \mathrm{h}$, with complex system calibration. Failure tire positioning is not unique enough, and strain values cannot be contemplated visually and appropriately. Gadget will automatically turn on alarm while tire pressure is too low or there's leakage. Motive force can intuitively understand every tire pressure circumstance thru direct-kind tpms.In assessment, direct kind tpms is superior to oblique-kind tpms in feature and overall performance, so this system used the direct- kind tpms.It specifically consisted of wi-fi tire pressure monitoring modules connected in tire. Wi-fi tire-pressure tracking modules real- well timed measured tire pressure, temperature and the measured signals had been transmitted after modulated into excessive frequency signals. Important receiving module acquired the alerts and displayed the received tire pressure, temperature data on the screen for motive force to consult. If tire strain and temperature abnormalities arise, then imperative receiving module will provide relevant alarm signal to remind driving force to take necessary measures. Gadget block diagram is proven in determine.The tool proposed right here is straight-tpms. A right away tpms particularly collected of additives i.e. The transmitter unit moreover referred to as strains monitoring module and the receiver util.Strain show module includes strain detectors, temperature sensor, onchip computer unit and wireless frequency transceiver chip. The receiver apparatus includes microcontroller unit, rf transceiver chip, liquid crystal show display or tft screen and the buzzer circuit.

\section{2] Block diagram consist of:}

Pressure sensor: To sense strain of the tire. Temperature sensor: to feel temperature of the tire.

ADC(analog to virtual converter): to transform analog.

Microcontroller: to virtually take delivery of data from the sensors, control it and transfer it serially to wi-fi transceiver. Wi-fi transceiver: to talk wirelessly with the receiver unit.

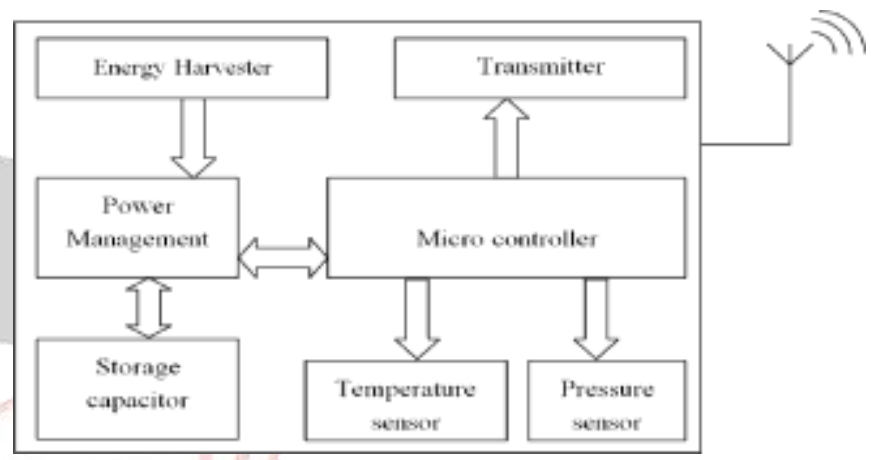

Fig 1:- Block Diagram of system

3.3] Component List \& Description:
A. Pressure Sensor.
B. Temperature Sensor.
C. Display (LCD).
D. ZigBee.
E. Energy Deliver.

A] Pressure sensor:

A strain sensor is a tool for stress measurement of gasoline or liquor. Stress is type of the potency essential for forestall a liquor from increasing, and is generally expressed in terms of pressure individual segment vicinity. A strain detector commonly take measure as a transducer, it cause a sign as a feature of the strain foist. For the functions of this newsletter, one of these sign is electric.

Stress detectors are cast to govern and observe in hundreds of day to day scenario. Strain detectors also applicable in a roundabout way degree particular variables which includes fluid/fuel drift, pace, water diploma, and altitude.

Strain detectors can vary substantially in generation, format, basic overall achievement, practical case scenario suitability and cost.

There can be additionally a class of strain sensors which is probably plan to degree in a energetic manner for shooting very excessive velocity differs in strain.Example practical case scenario for this form of detectors are likely within the 
quantify of burning strain in an machine barrel or in a gas turbine.

Those detectors are normally artificial out of piezoelectric substances which include quartz.

A few stress detectors are strain switches, which turn on or off at a selected pressure. As an instance, a water pump can be managed by a stress transfer in order that it starts off evolved while aqua is launched from the device, diminishing the strain in a reservoir.

\section{B] Temperature sensor:}

A temperature detector is a tool, normally a resistance temperature detector gather the facts approximately temperature through a specific origin and transform information in comprehensible shape for a tool or monitor hotness detector are applied in plenty of programs such as air conditioner surrounding controls, meals undertaking devices, medical gadgets, synthetic managing and vehicle underneath the cowl observing and managing structures, and lots of others.

The RTD: A hotness detecting gauges whose resistance adjustments with hotness. Normally made up of platinum, despite the reality that devices crafted using $\mathrm{Ni}$ or $\mathrm{Cu}$ are common place, resistive hotness detectors are able to have many top notch structure such as twine cut, skinny movie. To diploma the opposing finally of resistance hotness detector, workout a normal current, diploma the ensuing voltage, and dictate the resistance hotness detectors. The pt100 rtd assessment board uses ground arrangement rtd to diploma temperature.Pt100 additionally may be linked with degree hotness in far off regions. The temperature detector mentioned above are prejudice the usage of a regular present day deliver. So that you can reduce self-warmth because of strength dissipation, the cutting- edge significance is somewhat low.Circuit proven in determines is consistent contemporary supply uses a recommendation voltage, single amplifier, and a pnp transistor.

\section{C] LCD:}

Liquid crystal display (liquid crystal display) is a kind of horizontal panel unveil which uses liquid crystals in its top most position as per functionality. Liquid crystal show's to be had tremendous and ranging set of use cases for customers and businesses, as they'll be commonly placed in phones, televisions, laptop video show gadgets and device panels.

Lcd's have been a huge leap in terms of the era they changed, which encompass light-emitting diode (led) and gasoline-plasma indicates. Lcd's sanctioned presentations to be a plenty narrow than cathode ray tube (crt) generation. Lcd's utilizes tons considerable fewer electricity than led and fuel-display due to the fact they paintings on the principle of blocking off light in desire to emitting it. Wherein an led emits light,liquid crystal display produces an photograph the use of a backlight.

$$
\text { D] Zigbee: }
$$

The technology is meant to be less difficult and less expensive than different wpans, including bluetooth. Zigbee is centered at radio-frequency (rf) applications which require a low data fee, lengthy battery lifestyles, and secure networking.

Zigbee is an ieee 802.15.Four-primarily depends completely description for a fixed of immoderate-level transmission treaty accustomed to create non-public region networks, diminished potential binary wireless transmission, which includes for place artificialintelligence, clinical systems information gathering, and particular less potential, short distance needs, designed for small scale responsibilities which require wi-fi connection. For that reason, zigbee is a low- strength, low facts charge, and adjacent accessibility(i.e., private area) $\mathrm{Wi}-\mathrm{Fi}$ ad hoc community.

The era described with the aid of the use of the zigbee specification is supposed to be less hard and much less high priced than exclusive wi-fi personal location networks (wpans), as a example bluetooth or greater popular wi-fi networking such as wi-fi. Practical use encompass wireless moderate lever, domestic electricity video display units, visitors' management structures, and special customer and commercial tool that calls for quick-variety low-charge wifi data switch.

Its low electricity intake limits transmission distances to ten-a hundred meters sightline, relying on power outtern and surrounding traits. Zigbee gadgets can pass on data on top of extended interval by using passing records through a mesh community of halfway apparatus to acquire additional far off ones. Zigbee is commonly carried out in short data packages that require lengthen accumulator existence and cozy hobnob.

\section{E] Energy Deliver:}

Strength is the spine of any computerized gadget and the strength deliver is what feeds the appliance. Deciding on the right deliver may be the important distinction within a tool operating at premiere ranges and one which could carry irregular outcome.

Similarly to rotating present day (ac) to undeviating current (dc) electricity elements, direct current to direct current transformer also are available. If direct current is so far to be had for your appliance, a direct current to direct current change can be the better layout option than ac talk about beneath.

\section{4] SOFTWARE DESIGN:}

We are using here embedded c programming language. Embedded $\mathrm{c}$ is a powerful, feature rich development device for any on-chip computer. It's miles designed to offer the 
programmer with the very best viable solution for growing applications without compromising overall performance or manage.

\section{5] FLOWCHART:}

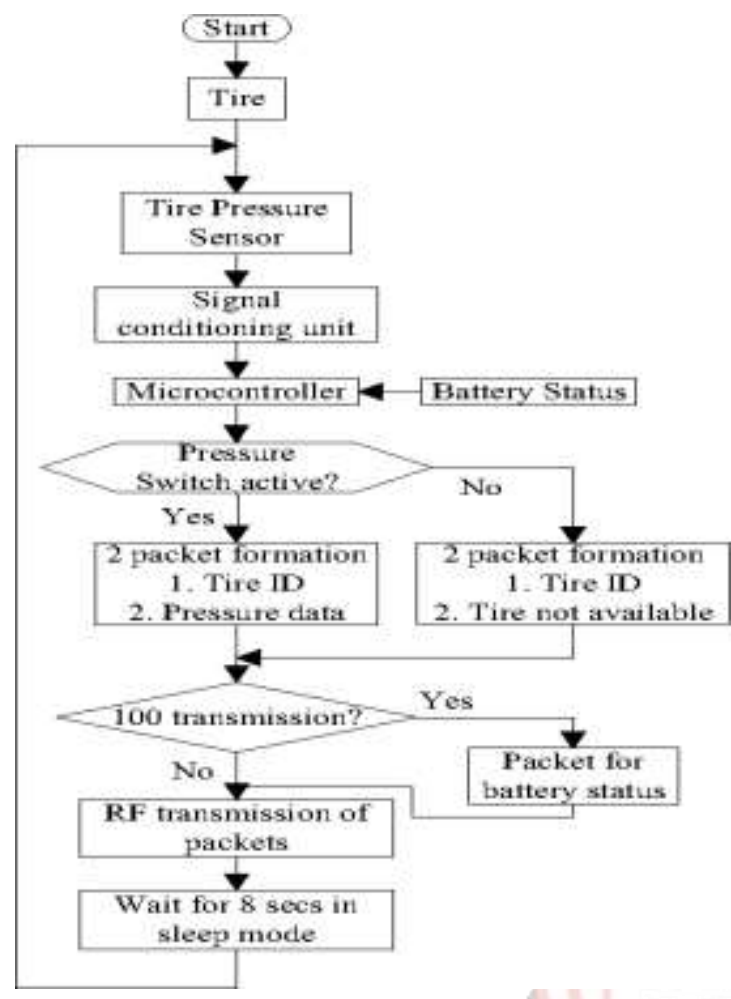

Fig 2: Flow-Chart

\section{CONCLUSION}

This project allows the rider to continuously monitor the tire strain which ends up in lessen gasoline productivity and rarely injuries,because of diminished strain tires. For this reason our idea is at once conveying the element of growing combustible performance and guaranteed renovation. This manner can be carried out nearly in each vehicles gift because of the reality observing strain is of predominant importance integrating functions of every equipment additives used have been evolved in it.Existence of each and every module has been logical out and located cautiously, therefore contributing to the high-quality working of the unit. Secondly, the use of noticeably superior integrated circuits with the help of growing technical automation, the assignment has been correctly carried out. Accordingly the undertaking has been correctly designed and examined.

\section{1] ACKNOWLEDEMENT:}

We express our sincere gratitude to our institute Sandip Foundation (SITRC), Nashik. We are thankful to the almighty for giving me the opportunity for carrying out this work under the guidance of Prof (Dr.) Gayatri M. Phade. Her encouragement and teaching have helped me to grow intellectually in a truly efficient manner.

\section{REFERENCES}

[1] Arun Kumar N., Srinivasan V., Krishna Kumar P., Analysing the strength of unidirectional fibre orientations under transverse static load, International Journal of Applied Engineering Research, v-9, i22, pp- 7749-7754, 2014.

[2] Srinivasan V., Analysis of static and dynamic load on hydrostatic bearing with variable viscosity and pressure, Indian Journal of Science and Technology, v- 6, i-SUPPL.6, pp-4777- 4782, 2013.

[3] Srinivasan V., Optimizing air traffic conflict and congestion using genetic algorithm, Middle - East Journal of Scientific Research, v-20, i-4, pp-456- 461, 2014.

[4] Praveen R. Achudhan M., Optimization of jute composite as a noise retardant material, International Journal of Applied Engineering Research, v- 9, i-22, pp- 7627-7632, 2014.

[5] Raja Kumar G., Achudhan M., Srinivasa Rao G., Studies on corrosion behaviour of borated stainless steel (304B) welds, International Journal of Applied Engineering Research, v-9, i- 22, pp-7767-7772, 2014.

[6] Ganeshram V. Achudhan M., Design and moldflow analysis of piston cooling nozzle in automobiles, Indian Journal of Science and Technology, v- 6, i- SUPPL.6, pp-4808-4813, 2013.

[7] Prof. Dr. G .M .Phade, “A Novel ICT Tool For Interactive Learning For Electronics Engineering Based On Augmented Reality", International journal of scientific and technology research (IJSTR) ISSN: 2277- 8616, Vol 8 Issue 08,AUGUST 2019.

[8] Prof. Dr. G .M .Phade, "Embedded Vision Based Cost Effective Teleoperating Smart Robot", International Journal of Innovative Technology and Exploring Engineering (IJITEE), ISSN: 2278-3075, Volume-8 Issue-7,pp.1544-1550 May, 2019,5.54,IJITEE Scopus, Elsevier.

[9] Prof. Dr. G.M .Phade, "Leap Motion Estimation for Controlling and tracking of drone", Sandip Foundation's International Journal on Emerging Trends in Technology (IJETT), ISSN:2455-0124, Vol.6, pp.12016-12019, April 2019, IJETT, Google scholar.

[10] Ganeshram V. Achudhan M., Synthesis and characterization of phenolrmaldehyde resin as a binder used for coated abrasives, Indian Journal of Science and Technology, v- 6, i-SUPPL.6, pp-4814-4823, 2013.

[11] Achudhan M., Prem,Jayakumar M., Mathematical modeling and control of an electrically-heated catalyst, International Journal of Applied Engineering Research, v- 9, i-23, pp- 23013-, 2014.

[12] Anbazhagan R., Satheesh B., Gopalakrishnan K., Mathematical modeling and simulation of modern cars in the role of stability analysis, Indian Journal of Science and Technology, v-6, i-SUPPL5, pp-4633- 4641, 2013.

[13] Loya Chandreshkumar, Joshi Pranav, Chaudhari Hemraj, Prof. Gayatri Bokade "Tyre Pressure Monitoring System And Fuel Leak Detection" International Journal of Engineering Research and Applications (IJERA) ISSN: 2248-9622, Vol. 3, Issue 3, May-Jun 2013, pp.345- 348.

[14] Jagdish Patel, "FPGA Based Efficient Implementation of Viterbi Decoder" in International Journal of Engineering and Advanced Technology (IJEAT), pp 84- 89, Oct 2011, ISSN: 2249-8958.

[15] Jagdish Patel. "War Field Robot Controlled By Android Phone" in International Journal of Innovative Research in Computer and Communication Engineering (IJIRCCE), pp.47 to 51,January 2015, ISSN: 2320- 9801.

[16] Jagdish Patel, "A Research paper on Manual Fixture Automation using PLC" in International Journal for Scientific Research \&amp; Development (IJSRD), pp.1705-1707,April 2017, ISSN: 2321-0613. 